\title{
Large-scale magnetic fields and anomalies of chemical composition of stellar coronae
}

\author{
V.V. Pipin and V.M. Tomozov \\ Institute Solar-Terrestrial Physics, Irkutsk
}

\begin{abstract}
We present evidence that anomalies in abundance of the chemical admixtures with the low first ionization potential (FIP) in the low corona of the late-type stars can be related to a topology of the large-scale magnetic field. Observational data from Ulysses and the Stanford Solar Observatory reveal the high correlations between the FIP effect of the solar wind and amount of the open magnetic flux. To analyze the stellar activity data we relate the amount of the open magnetic flux with the ratio between poloidal and toroidal magnetic field of a star. The solar-type stars show the increase abundance of the low FIP elements relative to elements with the high FIP with the decrease of the large-scale poloidal magnetic field (and increase the toroidal component of the magnetic field). The branch of the fully convective stars demonstrates inversion of the FIP-effect. This inversion can result from strong coronal activity, which is followed by the strong poloidal magnetic on these stars.
\end{abstract}

Keywords: Sun-corona; solar-stellar analogy; magnetic fields; FIP effect 


\section{Introduction}

Observations show that the stellar magnetic activity depends largely on the stellar mass and the rotational period (see, Donati and Landstreet, 2009; Vidotto et al., 2014: See et al., 2016). These parameters determine a level of the magnetic activity (Noyes et al., 1984) and the typical topology of the large-scale magnetic field (Donati et al. 2008a| See et al. 2016). Therefore, we can, generally, expect that the chromospheric and coronal activity in late-type stars varies with the spectral class and rotational period, correspondingly (Reiners, 2012).

It is widely accepted that chemical composition in the solar photosphere is spatially uniform. On another hand, Pottasch (1963) showed that the coronal chemical composition can depend on the first ionization potential (hereafter FIP). Moreover, elements with low FIP $(\leq 10 \mathrm{eV})$, for example, $\mathrm{Fe}, \mathrm{Mg}, \mathrm{Si}$, $\mathrm{K}$ etc, show an increase of abundances in the coronal loops above the active regions. At the same time, elements with high FIP $(\mathrm{FIP}>10 \mathrm{eV})$ show no variation or, sometimes, even a little-decreased composition there. This is socalled 'FIP-effect'. General information about manifestations of FIP effect in solar atmosphere, solar wind and in composition of solar energetic particles are presented in Meyer's review (1985).

Observations showed that elements with low FIP can be accumulated at top of the closed magnetic loops in course of the active regions evolution. For example, Widing and Feldman (2001) found that chemical composition of the coronal plasma above recently emerging active region is close to the composition at the photosphere, and after that, it shows increased concentrations of the elements with low FIP at the top of a coronal loop. The ratio between the coronal and photospheric abundances of low FIP elements can reach factor 4 after few days of the active region evolution. Also, they found that in the long-lived active regions the increasing concentration of the elements with low FIP can reach factor 8 or even higher than that value. Observations show complicated distributions of the low FIP admixtures in the coronal plasma above the active regions. Recently, new interesting results have been obtained on FIP effect evolution and distribution in solar active structures. Using results of observations of EIS spectrometer onboard Hinode, Baker et al. (2015) found that the low FIP element enrichment occurs in upper parts of coronal loops located in central zones of the active region. At the active region periphery, a process of magnetic reconnection with emerging small-scale magnetic fields with photospheric plasma composition neutralizes accumulation of the low FIP elements. As a result, the mixed plasma composition with reduced FIP bias is transferred by interchange reconnection beyond active region boundaries and can manifest itself in slow wind composition.

The detailed observations have shown that element composition in a hot plasma, which evaporated during large X-ray flares turned out to be close to the composition of the photosphere (Warren, 2014). At the same time, plasma composition in CME events connected with of such flares was heavily enriched of low FIP elements with a high ionization degree (Zurbuchen et al., 2016). Additionally, with EIS spectrometer on Hinode measurements was made of the 
plasma composition during impulsive heating events in the solar transition region. During these events, spectral lines of high FIP elements were amplified with respect to low FIP lines and showed a composition close to photospheric one. On the contrary, overlying long-lived coronal fan loops showed low FIP element enrichment (Warren et al. 2016). These authors concluded that plasma composition is an important feature of the coronal heating process, with impulsive heating (such as a flare or coronal ejection) leading to evaporation of photospheric plasma; and in higher frequency heating (for example, wave heating) resulting in enrichment low FIP in the long-lived coronal structures.

The above consideration shows that the FIP fractionation of the solar coronal plasma is a rather complicated process. The key questions for us are as follows. Firstly, is the FIP fractionation controlled by the global parameters of the solar activity? Secondly, what are those global parameters of the magnetic activity that determine the magnitude of the FIP-effect for the Sun as a star? These questions are motivated by the solar and stellar observations. Using observational data from SDO Brooks et al. (2017) showed that variations of the integral FIP-effect for the Sun as a star is correlated with a proxy for solar activity, the F10.7 radio flux, and therefore, with the solar cycle phase. Exploring the late-type stars, Wood et al. (2012) found a systematic decrease of the FIP effect with the stellar mass. Moreover, their results show an inversion of the FIP-effect for the low mass M-dwarfs (inversed FIP effect). Results of Wood et al. (2012) were confirmed by Brooks et al. (2017). The global parameters of the stellar magnetic activity changes with the stellar mass (Donati \& Landstreet 2009). From earlier studies (see, e.g., Skumanich 1972, Noyes et al. 1984) we know that another critical parameter for stellar magnetic activity is the stellar rotation rate.

Interesting that the results of Wood et al. (2012) do not show a relation between the coronal FIP effect and coronal activity parameters such as the X-ray luminosity $\left(\log L_{X}\right)$. The same was found for the surface X-ray flux $\left(\log F_{X}\right)$. On another hand, these parameters show clear correlations with rotation rate and the Rossby number of a star (see, Pevtsov et al. 2003; Wright and Drake 2016). This motivate us to explore the connection of the coronal chemical composition with other parameters of the stellar magnetic activity. One of the most important global parameter of the stellar coronal field is an amount of the open and closed magnetic flux. This parameter is tightly related with the strength of the stellar wind and the magnetic braking of the angular momentum of a star (Mestel, 1968).

Fletcher et al. (2015) pointed out that chemical composition of plasma of the slow component of the solar wind shows an increase concentration of elements with low FIP by factor 3 compares to their values for the fast component of the solar wind. Woo et al. (2004) showed that the so-called "slow" and "fast" components of the solar wind can be related to the closed and open magnetic flux of the Sun. This is employed in the solar wind models (Wang, 2012). Note, that in the global models of the solar coronal field the definition of the open and closed magnetic structure is phenomenological and it is related to the so-called "source surface" (Schatten et al. 1969). Laming (2004) employ 
the magnetic topology to model the solar coronal FIP effect. In his model, it is assumed that FIP fractionation is induced by the so-called ponderomotive force which results from propagation of the resonant alfvenic waves along the closed magnetic field lines. Direction and amplitude of the ponderomotive force depend on the gradient of wave energy in the low corona. For the resonant waves the energy grows up and this results in the upward ponderomotive force acting on ions of the low FIP. In the open magnetic field structures and for the off-resonant waves the wave energy decreases in the major part of the low corona. In following to ideas of Woo et al. (2004) and Laming (2004) let us assume that the average FIP effect of the Sun as a star approximately reflects the ratio between amounts of the open and closed fluxes of the solar coronal magnetic field. In our analysis we have to keep in mind that the open field configurations can have a slow wind composition and closed field can have a photospheric composition. This may be a source of systematic error in our study.

The purpose of the paper to study the statistical relationships between the global parameters of the stellar magnetic activity such as of the open and closed magnetic flux and the integral parameter of the coronal FIP effect. Both the solar and stellar activity observations data will be discussed. In our study, we use a combination of data from results of Wood et al. (2012) and other data sets including the solar wind composition from the Ulysses space mission, the magnetic field observations from the Wilcox Solar Observatory, the stellar magnetic field spectropolarimetric measurements (see, Petit et al., 2014 and Marsden et al., 2014). In next section, we will describe the datasets, which will be used in this paper.

\section{Data}

\subsection{Solar observations}

To trace solar cycle variations of the FIP effect of the Sun as a star we use observations of Ulysses spacescraft. The data from instrument Ulysses/SWICS ( Solar Wind Ion Composition Spectrometer) are used. It is assumed that insitu measurements of the wind's composition of heavy ions, e.g., such as $\mathrm{O}$, $\mathrm{C}$, and $\mathrm{Fe}$, can be used to infer its composition in the low corona (Buergi and Geiss, 1986). In this paper we will use the SWICS's measurements for ions of Fe and $\mathrm{O}$. The measurements procedure and their implications were described in details by Geiss et al. (1995) and von Steiger et al. (1995). The observed ratio $(\mathrm{Fe} / \mathrm{O})$ gives a proxy for the magnitude of the FIP fractionation in the solar corona. The magnitude of the FIP effect is defined as follows:

$$
\mathrm{FIP}=\frac{[\mathrm{Fe} / \mathrm{O}]_{\mathrm{c}}}{[\mathrm{Fe} / \mathrm{O}]_{p h}} .
$$

Following to procedure given by Wood et al. (2012) and Laming (2015) we introduce the so-called "FIP bias". It is determined by ratio of the photospheric 


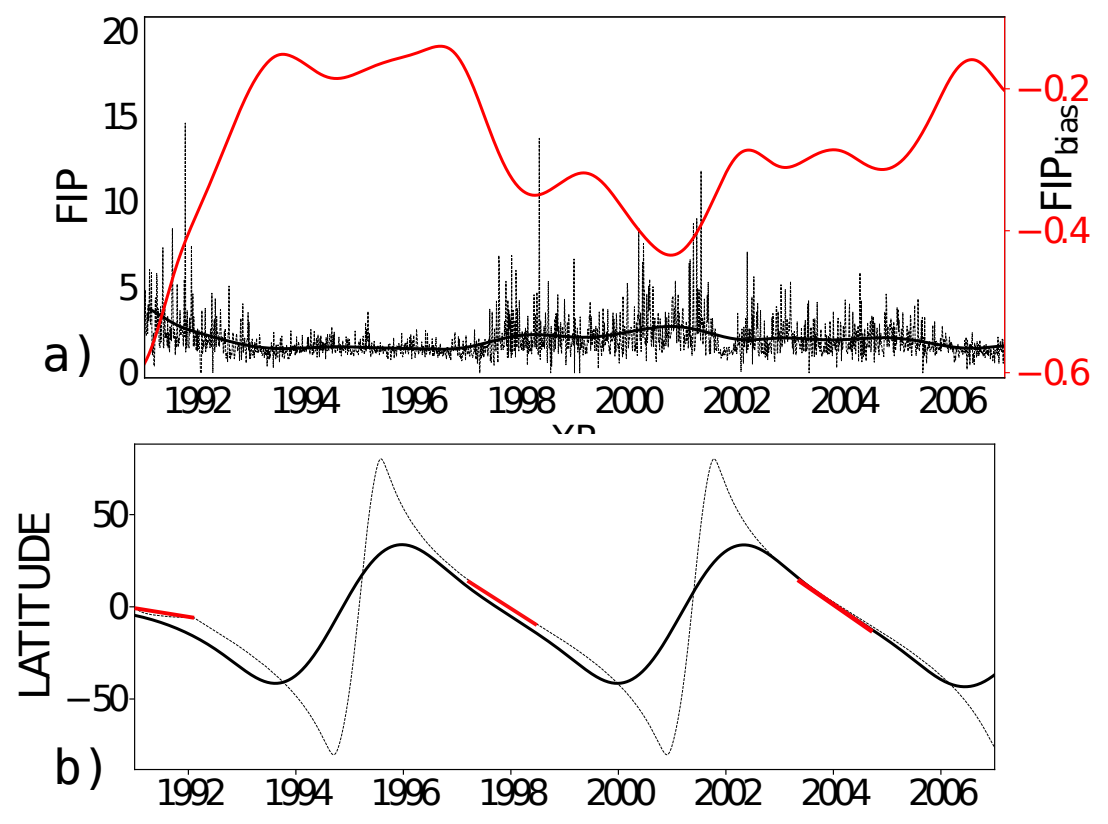

Figure 1: a) The FIP fractionation (see, Eq.1) from the Ulysses/SWICS, the original data (dashed curve, 3-days cadence) and after the Gaussian filtering (the solid curve). The red line curves show the FIP bias (see, Eq.2) with magnitude shown by the right y-axis; b) Variations of the helio-latitude of the ULYSSES spacecraft (dotted curve). The solid curve shows the "effective" latitude of the observations after smoothing. The red patches show the separated intervals for analysis (see, the main text).

value of $\mathrm{Fe} / \mathrm{O}$ and its coronal counterpart, i.e,

$$
\mathrm{FIP}_{\text {bias }}=\log [\mathrm{Fe} / \mathrm{O}]_{p h}-\log [\mathrm{Fe} / \mathrm{O}]_{c} .
$$

Therefore, we have $\mathrm{FIP}_{\text {bias }}=-\log$ FIP. Hereafter, we assume that $\log [\mathrm{Fe} / \mathrm{O}]_{p h}=$ 0.06 (Laming, 2015). The relative error of the SWICS's measurements of the elemental composition is estimated to be 10- 25\% (see, e.g., Geiss et al., 1995).

Superposed epoch analysis of Geiss et al., 1995 showed the rather tight correlation between the magnitude of FIP fractionation and coronal structures related to regions of the fast and slow solar wind. Their results clearly showed that FIP fractionation is small in coronal holes. These solar phenomena are usually related to the open magnetic field structures. On another hand, the FIP fractionation grows by factor 3-4 above the active regions, where the closed magnetic field structures dominate. This conclusion was further stressed by Fletcher et al. (2015).

The trajectory of the Ulysses spacecraft passed over very different parts of the solar disk. To deduce the FIP effect for the Sun as a star we smoothed the data by the Gaussian filter with the FWHM equal one year. The result is shown in Figure 1. The graph shows that in the smoothed data the FIP fractionation had a maximum magnitude of factor 4 . It occurs near the maximum of the 
22 solar cycle. The corresponded value of FIP bias was about -0.6. Laming (2015) and Brooks et al. (2017) argued that the maximum FIP bias for the Sun as a star during epochs of solar maxims should be around -0.6. However, their results are related to cycles 20 and 24 . Those cycles have the considerable smaller magnitudes than cycles 22 and 23. For the minimum of cycle 23 Brooks et al. (2017) found $\mathrm{FIP}_{\text {bias }} \approx-0.16$. This roughly agrees with our estimation from the smoothed data.

The aim of the smoothing procedure is to get an estimation of the FIP effect of the Sun as a star. Another purpose is to eliminate the possible correlation between the measured $\mathrm{Fe} / \mathrm{O}$ ratio and the spacecraft latitude (see Figures $1 \mathrm{a}$ and b). However, we must take into account the fact that the latitude of the spacecraft's flight around the Sun varied non-monotonically, and, in some periods, the trajectory passed above equatorial latitudes during a long time (more than one year). As a result, the applied smoothing procedure may yield a distorted estimate of the Sun as a star's FIP effect, in spite of averaging over a large number of solar rotation. To analyze this in detail we look at those particular periods of times. The data sets include the following time intervals: 1991-1992, 1997-1999 and 2003-2005. The corresponded patches are marked by red color, see Figure1 $\mathrm{b}$. For these periods of time the original Ulysses data were averaged on the interval of the one Carrington rotation. In this case, the averaged FIP effect represent contributions from the equatorial part of the Sun.

Parameters of the solar magnetic field were determined using observations of the Wilcox Solar Observatory (WSO), see Duvall et al. (1979) and Hoeksema (1995). These data span about four solar cycles starting from 1976. The large-scale magnetic field was restored using the spherical harmonic coefficients obtained from the potential field extrapolation employing the so-called "source surface" at $2.5 \mathrm{R}$. More details about the procedure to determine the spherical harmonic coefficients decomposition can be found at the WSO website. Using the obtained distributions of the large-scale magnetic field we determine the unsigned open, $\Phi_{o}(t)$, the closed,$\Phi_{c}(t)$, and the total, $\Phi_{s}(t)$, magnetic fluxes as follows

$$
\begin{aligned}
\Phi_{o}(t) & =R_{s}^{2} \int\left|B_{r}(\theta, \phi, t)\right|_{r=R_{s}} \sin \theta d \phi d \theta, \\
\Phi_{s}(t) & =R^{2} \int\left|B_{r}(\theta, \phi, t)\right|_{r=R} \sin \theta d \phi d \theta \\
\Phi_{c}(t) & =\Phi_{s}(t)-\Phi_{o}(t)
\end{aligned}
$$

where $\phi$ is longitude, $\theta$ is the polar angle and the source surface radius is $R_{s}=2.5 R$. Also we need a proxy for the ratio $\Phi_{o}(t) /\left(\Phi_{s}(t)\right)$. For this we suggest to employ the mean magnitudes of the axisymmetric poloidal and total (axisymmetric and nonaxisymmetric) toroidal magnetic field, which are as follows, $\overline{B^{(P A)}}=\left\langle\sqrt{B_{r}^{2}(\theta, t)+B_{\theta}^{2}(\theta, t)}\right\rangle_{r=R}$, and $\overline{B^{(T)}}=\left.\overline{\mid B_{\phi}(\theta, \phi, t)}\right|_{r=R}$, where the magnetic field components are inferred from the same spherical harmonic coefficients. We introduce another parameter as a proxy for the ratio 


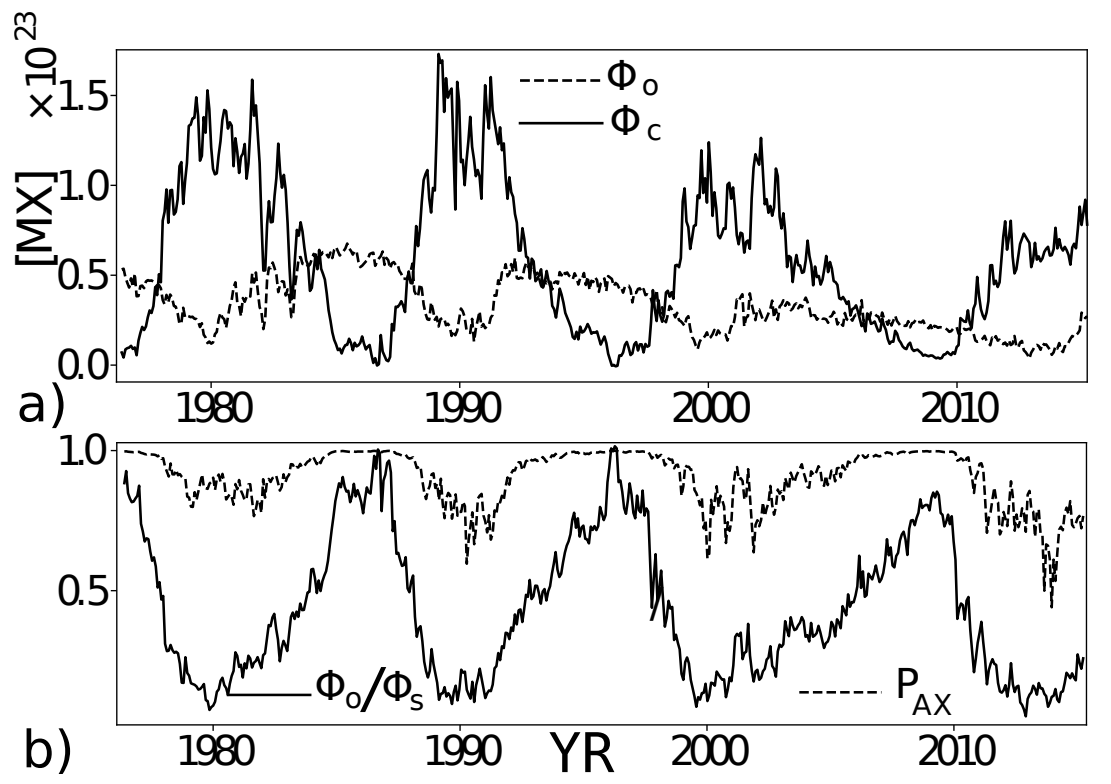

Figure 2: a) The unsigned open and closed magnetic fluxes deduced from the WSO's harmonic coefficients; b) ratio $\Phi_{o}(t) /\left(\Phi_{s}(t)\right)$ (solid black line); the dashed line shows the parameter $\mathrm{P}_{\mathrm{AX}}$ (the relative contribution of the axisymmetric poloidal field, see E6). The points on the graphs represent the individual Carrington rotations.

$\Phi_{o}(t) /\left(\Phi_{s}(t)\right):$

$$
P_{A X}=\frac{\overline{B^{(P A)}}}{\overline{B^{(P A)}}+\overline{B^{(T)}}} .
$$

The reason to express the relative contribution of the axisymmetric poloidal magnetic field in form of $\mathrm{Eq}(6)$ is that it correlates well with $\Phi_{o} / \Phi_{s}$. Figure 2 shows results for the defined parameters of the solar magnetic field. The points on the graph represent the individual Carrington rotations. The given time profiles of the parameters $\Phi_{o}(t) /\left(\Phi_{s}(t)\right), \Phi_{c}(t), \mathrm{P}_{\mathrm{AX}}$ and $\overline{B^{(T)}}$ were further smoothed with the same Gaussian filter as the data about chemical composition. Note, that the $\overline{B^{(T)}}$ represent the non-axisymmetric toroidal magnetic field on the solar surface.

We have no estimations for the total magnetic flux parameters for our sample of stars. For this purpose, we will employ $\mathrm{P}_{\mathrm{AX}}$ ( the relative contribution of the axisymmetric poloidal field) as a proxy of $\Phi_{o} / \Phi_{s}$, and the parameter $\overline{B^{(T)}}$ (the mean strength of the nonaxisymmetric toroidal magnetic field) is a proxy for the unsigned total closed flux $\Phi_{c}$. Figures 3 a and b show that our choice is reasonable. In both cases, we found the tight relations in point to point correlations and in the smoothed time-series, as well. The relation of $\Phi_{o} / \Phi_{s}$ and $\mathrm{P}_{\mathrm{AX}}$ seems to be a power law. The best approximation is as follows: $\Phi_{o} / \Phi_{s} \sim \mathrm{P}_{\mathrm{AX}}{ }^{4}$. On another hand, $\Phi_{c}$ and $\overline{B^{(T)}}$ are linearly connected. 

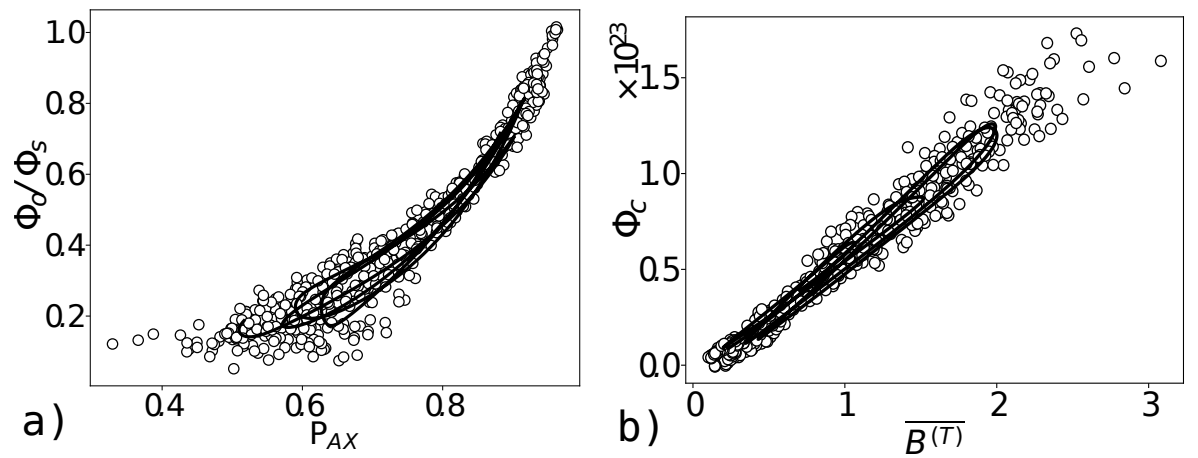

Figure 3: a) Relation between parameters $\mathrm{P}_{\mathrm{AX}}$ and $\Phi_{o} / \Phi_{s}$ for the individual rotations (circles) and the smoothed time series (solid line); b) the same as (a) for $\Phi_{c}$ vs $\overline{B^{(T)}}$.

\begin{tabular}{ccccc}
\hline & $\mathrm{s}$ & $\mathrm{p}$ & $\mathrm{r}$ & $\sigma$ \\
\hline$\Phi_{o} / \Phi_{s} \sim \mathrm{P}_{\mathrm{AX}}{ }^{4}$ & 1.02 & 0.0 & 0.98 & 0.01 \\
\hline$\Phi_{c} \sim \overline{B^{(T)}}$ & $6.44 \cdot 10^{22} \frac{\mathrm{Mx}}{\mathrm{G}}$ & 0.0 & 0.97 & $5.89 \cdot 10^{20}$ \\
\hline
\end{tabular}

Table 1: Parameters of the linear regressions between the solar magnetic flux and the mean parameters of the magnetic components: $\mathrm{s}$ is a slope of the regression line, $\mathrm{p}$ is two-sided significance of its deviation from zero, $\mathrm{r}$ is a correlation coefficient, and $\sigma$ is the standard error of the estimate.

Parameters of the linear regressions for $\Phi_{o} / \Phi_{s} \sim \mathrm{P}_{\mathrm{AX}}{ }^{4}$ and $\Phi_{c} \sim \overline{B^{(T)}}$ are given in Table1. In both cases the correlation coefficients are about 0.98. Estimations of the Spearman's rank correlations give the similar correlations coefficients, i.e., $\mathrm{r}=0.975$ for $\Phi_{o} / \Phi_{s} \sim \mathrm{P}_{\mathrm{Ax}}{ }^{4}$ and $\mathrm{r}=0.967$ for $\Phi_{c} \sim \overline{B^{(T)}}$. Using these results we will try to connect the stellar FIP $_{\text {bias }}$ observation with magnetic parameters $\mathrm{P}_{\mathrm{AX}}$ and $\overline{B^{(T)}}$.

\subsection{Stellar magnetic observations}

Table 2 gives the list of the stars included in our analysis. The set covers a range of spectral classes from the mid $\mathrm{G}$ to the mid-M dwarfs. The color index parameter B-V is taken from the survey of Marsden et al. (2014).

Inferring parameters of magnetic activity of the fast rotating stars became possible after implementation of Zeeman-Doppler Imaging method (see, Donati and Brown, 1997, Donati, 2001; Donati and Landstreet, 2009). This method is good for determination of the large-scale magnetic field components of stars with a fast rotation rate. This condition makes possible to isolate the rotational dynamics of the Zeeman components in the background spectrum, that is subjected to the Doppler broadening. All stars in our set are rotating faster than the Sun, for example, $\epsilon$ Eri and $\xi$ Boo B have the rotational period of ten days and other stars are rotating faster. They also have the higher magnetic activity. 


\begin{tabular}{|c|c|c|c|c|c|c|c|c|}
\hline & B-V & $\log \frac{\bar{B}^{2}}{[G]^{2}}$ & $\overline{B^{(T)}},[\mathrm{G}]$ & $\overline{B^{(P)}},[\mathrm{G}]$ & $\overline{B^{(P A)}},[\mathrm{G}]$ & $\mathrm{FIP}_{\text {bias }}$ & $\mathrm{P}_{c y c}$ & Ref \\
\hline \multirow[t]{4}{*}{$\chi^{1}$ Ori } & 0.59 & 2.35 & 4.6 & 8.6 & 6.6 & -0.555 & $16.8, \mathrm{~L}$ & {$[1,2,5]$} \\
\hline & & 2.22 & 4.2 & 5.4 & 4.4 & & & \\
\hline & & 2.60 & 10.2 & 7.8 & 6.8 & & & \\
\hline & & 2.41 & 5.0 & 7.2 & 6.1 & & & \\
\hline \multirow[t]{2}{*}{ EK Dra } & 0.61 & 3.63 & 22.4 & 19.2 & 17.8 & -0.277 & $9.2, \mathrm{~L}$ & $-/-$ \\
\hline & & 3.90 & 43.8 & 36.6 & 34.3 & & & \\
\hline$\pi^{1} \mathrm{Uma}$ & 0.62 & 2.76 & 7.4 & 9.8 & 8.9 & -0.645 & $2.1, \mathrm{~L}$ & $-/-$ \\
\hline Sun & 0.66 & 1.16 & 0.94 & 3.4 & 2.77 & -0.3 & 11 & $\begin{array}{c}\text { this } \\
\text { work, } \\
{[1,4,5]}\end{array}$ \\
\hline \multirow[t]{2}{*}{$\kappa^{1}$ Ceti } & 0.68 & 2.88 & 13.8 & 16.9 & 9.5 & -0.462 & $5.9, \mathrm{~L}$ & {$[1,2,3,5]$} \\
\hline & & 2.64 & 11 & 8.6 & 7.7 & & & \\
\hline$\xi \mathrm{Boo} \mathrm{A}$ & 0.72 & 3.67 & 10.7 & 29.8 & 9.42 & -0.32 & $\begin{array}{l}4-5, \\
11, \mathrm{~L}\end{array}$ & $\begin{array}{c}1, \\
3,5]\end{array}$ \\
\hline \multirow[t]{2}{*}{ AB Dor } & 0.86 & 4.92 & 117.2 & 244.7 & 73.1 & 0.488 & $18, \mathrm{~L}$ & {$[1,3,5]$} \\
\hline & & 4.72 & 80.5 & 206.2 & 54.5 & & & \\
\hline \multirow[t]{6}{*}{$\epsilon$ Eri } & 0.88 & 2.27 & 1.1 & 13.1 & 4.1 & -0.06 & $3,13, \mathrm{~L}$ & {$[1,3,5]$} \\
\hline & & 2.08 & 2. & 13.2 & 10.1 & & & \\
\hline & & 2.50 & 2.7 & 11.4 & 9.7 & & & \\
\hline & & 2.09 & 2.1 & 9.5 & 7.6 & & & \\
\hline & & 2.63 & 6.2 & 15.3 & 10 & & & \\
\hline & & 2.66 & 3.0 & 18.9 & 8.6 & & & \\
\hline$\xi$ Boo B & 1.16 & 2.60 & 4.9 & 16.5 & 8.1 & -0.18 & $4.3, \mathrm{~L}$ & {$[1,3,5]$} \\
\hline EV Lac & 1.36 & 5.54 & 92.3 & 573 & 306 & 0.474 & & {$[1,3]$} \\
\hline AD Leo & 1.54 & 4.76 & 31.3 & 237 & 230 & 0.536 & & {$[1,3]$} \\
\hline EQ Peg A & 1.58 & 5.31 & 131.9 & 423 & 357 & 0.450 & & {$[1,3]$} \\
\hline EQ Peg B & 1.7 & 5.35 & 51 & 468 & 451 & 0.417 & & {$[1,3]$} \\
\hline
\end{tabular}

Table 2: The $\mathrm{B}-\mathrm{V}$ is the stellar color index; $\bar{B}$ is the mean magnetic field strength on the stellar surface; $\overline{B^{(T)}}$ is the same for the non-axisymmetric toroidal magnetic field; $\overline{B^{(P)}}$ is the mean strength of the poloidal magnetic field on the stellar surface (both axisymmetric and nonaxisymmetric); $\overline{B^{(P)}}$ is the same for the axisymmetric poloidal magnetic field; FIP $_{\text {bias }}$ is a parameter of the coronal FIP effect; $\mathrm{P}_{c y c}$ is period of the stellar magnetic cycle, $\mathrm{L}$ means the long-term magnetic variability trend (see, Egeland 2017). The last column gives list our references for magnetic data and FIP bias is as follows: [1 Laming (2015); [2 Rosén et al. (2016); [3 See et al. (2015); [4 Brooks et al. (2017), [5] Egeland (2017) 


\begin{tabular}{|c|c|c|c|c|}
\hline & $\begin{array}{c}\text { Magnetic } \\
\text { observations }\end{array}$ & References & $\mathrm{FIP}_{\text {bias }}$ & References \\
\hline$\chi^{1}$ Ori & $\begin{array}{l}2007.1,2008.1 \\
2010.8,20111.9\end{array}$ & [1] & 2001 & {$[5]$} \\
\hline EK Dra & $2007.1,2012.1$ & $-/-$ & 2001 & $-/-$ \\
\hline$\pi^{1} \mathrm{Uma}$ & 2007.1 & $-/-$ & 2001 & $-/-$ \\
\hline$\kappa^{1}$ Ceti & $\begin{array}{l}2012.8, \\
2013.7\end{array}$ & $-/-$ & 2001 & $-/-$ \\
\hline$\xi$ Boo A & 2013.09 & {$[2]$} & 2007.5 & {$[6,7]$} \\
\hline ab Dor & $2001.12,2002.12$ & $-/-$ & 2001 & {$[8,9]$} \\
\hline$\epsilon$ Eri & $\begin{array}{c}2007.1,2008.1 \\
2010.1,2011.10 \\
2012.10,2013.09\end{array}$ & $-/-$ & 2001.3 & {$[6]$} \\
\hline$\xi$ Boo B & 2013.09 & {$[3]$} & 2007.5 & {$[6,7]$} \\
\hline EV Lac & $\begin{array}{c}2006.8, \\
2007.7,8 /\end{array}$ & {$[3,4]$} & 2001 & {$[8,9]$} \\
\hline AD Leo & $\begin{array}{c}2007.1,2007.2 \\
2008.1,2008.2\end{array}$ & $-/-$ & 2001 & [9] \\
\hline EQ Peg A & 2006.8 & $-/-$ & 2006.11 & $-/-$ \\
\hline EQ Peg B & 2006.8 & $-/-$ & 2006.11 & $-/-$ \\
\hline
\end{tabular}

Table 3: Stars and their epochs of the magnetic measurements and measurements of the FIP -effect. The list our references is as follows: [1 Rosén et al. (2016); [2 See et al. (2015); [3 See et al. (2016); [4] Donati et al. (2008b); [5 Telleschi et al. (2005); 6] Wood and Linsky (2006); [7. Wood and Linsky (2010); [8. Testa et al. (2004); [9]. Liefke et al. (2008). 
Interesting that the magnetic activity of late-type stars is undoubtedly related to the rotational period (Noyes et al., 1984, Baliunas et al., 1995).

For the fast rotating young suns (having $\mathrm{B}-\mathrm{V} \sim 0.6$ ) we used results of Rosén et al. (2016) who used archival observational data of the spectropolarimetric measurements collected in the PolarBase (Petit et al. 2014). Results for the K and M-dwarfs are inferred from the data set given by See et al. (2015). All magnetic parameters listed in Table 2 were calculated using data given in the above-cited papers. The proxy parameter $P_{A X}$ was computed in following the given data sets. Rosén et al. (2016) showed that typical error for the single measurement of the line-of-sight magnetic field can be about 50 percents. However, having results for the different rotational phases the error can be reduced substantially. In comparing results of inversions from stellar observations with results from solar magnetic field extrapolation we have to take into account that the WSO pipeline calculates the PFSS (potential field source surface) decomposition assuming that the axisymmetric toroidal magnetic field on the surface is zero, $\overline{B^{(T A)}}=0$. This may be incorrect (Pipin and Pevtsov, 2014). Also, this is different from procedure employed for studying large-scale magnetic field on the fast rotating stars (see, Donati and Brown, 1997; Donati, 2001; Donati and Landstreet, 2009, Jardine et al., 2017). Note, that Vidotto (2016) considered application of the general procedure, i.e., a combination of the PFSS method and a non-zero toroidal magnetic field at the surface, for the SDO magnetic field measurements.

The Table 2 contains results about the coronal chemical composition parameter FIP $_{\text {bias }}$. There we listed parameters given in review of Laming (2015) (hereafter L15). Recently, Brooks et al. (2017) (hereafter B17) re-analyzed the data given in L15 and found that the typical error of the spectrometric inference of the $\mathrm{FIP}_{\text {bias }}$ is about 10 to 20 percents. The given data show a correlation between spectral class and the FIP $_{\text {bias }}$. It was found by Wood et al. (2012) and it was discussed in details in L15 and B17. Number of stars in our study is smaller because not all the stars, which were analyzed in L15 and B17, have the magnetic field measurements. Also we have to take into account that epochs of observations for the magnetic and chemical composition measurements are very different. The Table 3 shows epochs of observations for each star. Having data from different epochs of observations can bias our conclusion. Therefore analysis of the solar cycle variations of the FIP bias are rather important for understanding the stellar observations. Some further details about the solar-cycle variation of the FIP effect and its relation with the solar magnetic activity were discussed in Pipin and Tomozov (2018).

\section{Results}

\subsection{Solar cycle variation of FIP effect}

Figures $4 \mathrm{a}, \mathrm{b}$ and c show relations between the smoothed time-series of $\Phi_{o} / \Phi_{s}$ , $\Phi_{c}$ and $\Phi_{s}$ and the $\mathrm{FIP}_{\text {bias }}$. All those quantities have the solar cycle variations. This results in the close relations between evolution curves of the solar magnetic 

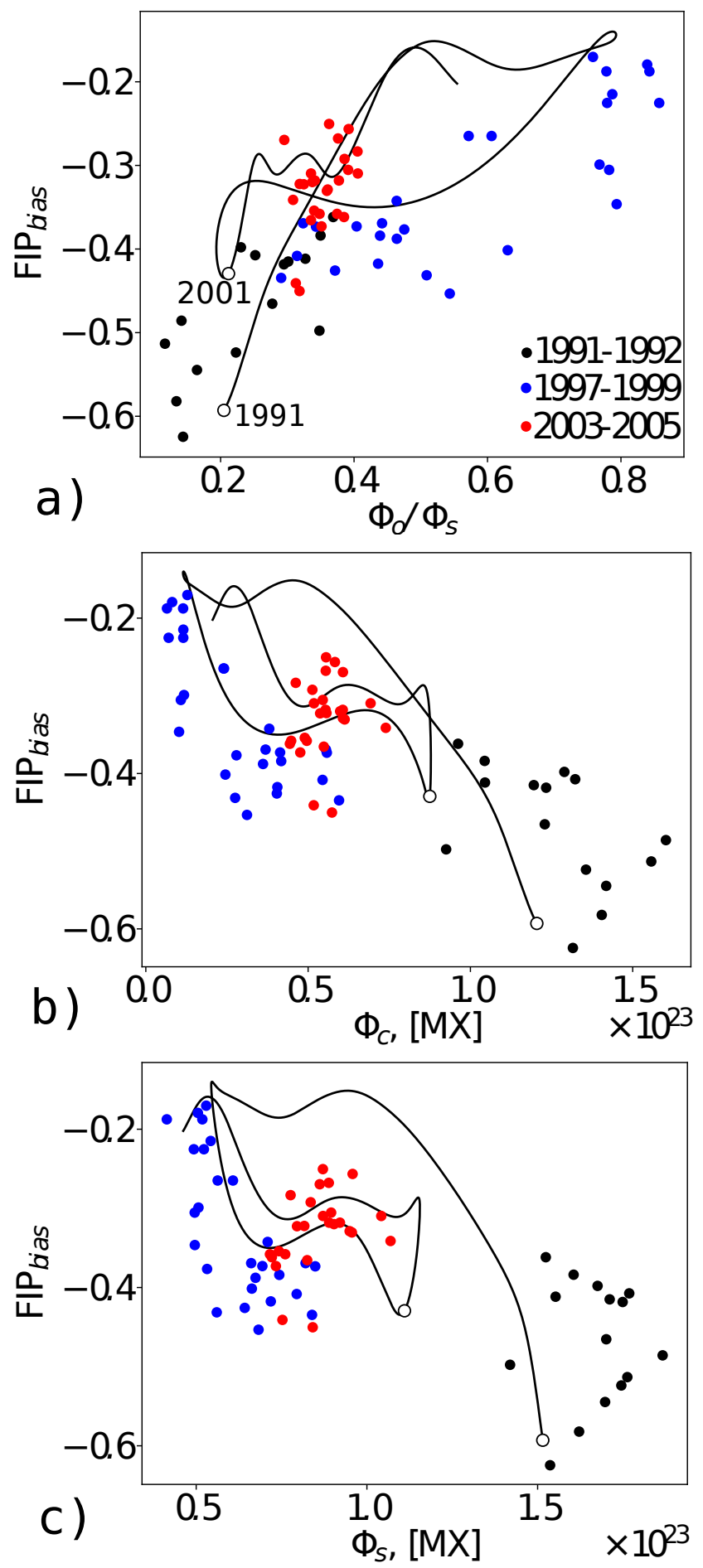

Figure 4: a) Solar cycle relation between the smoothed time-series of the magnetic fluxes ratio $\Phi_{o}(t) / \Phi_{s}(t)$ and the FIP $_{\text {bias }}$ (solid line) and pqint-to-point correlation between $\Phi_{o}(t) / \Phi_{s}(t)$ and the FIP bias $_{\text {for }}$ for individual Carrington rotations for the chosen periods of time (see Figure $1 \mathrm{p}$ and the main text); b) the same as (a) for c) the same as (a) for the total closed magnetic flux $\Phi_{c}(t)$; c) the same as (s) for the total surface magnetic flux $\Phi_{s}(t)$. The years 1991 and 2001 are marked by the white circles. 
flux parameters and the $\mathrm{FIP}_{\text {bias }}$ for the smoothed time series. The relations in the smoothed time-series do not necessarily mean the causal connections between the magnetic flux parameters and the FIP effect. To demonstrate this fact, Figure 4 shows correlations between the solar magnetic flux parameters and the FIP effect for the individual Carrington rotations during three particular periods of time when the latitude of the spacecraft was near equatorial regions and it was varying slowly (see, Figure 1 $\mathrm{b}$ ). In this case, the averaged (over one Carrington rotation) parameter $\mathrm{FIP}_{\text {bias }}$ represent contributions from the equatorial part of the Sun where the sunspot activity goes on. It is believed that sunspot activity is the major source of the solar FIP effect. Therefore the obtained parameter $\mathrm{FIP}_{\text {bias }}$ could be considered as a better representative for the integral FIP bias for the Sun as a star than that from the long-term smoothed time-series. The data sets include the following time intervals: 19911992, 1997-1999 and 2003-2005. The Table4 shows estimations of the Spearman rank correlations and the null hypothesis probability (that the datasets are uncorrelated). It is found that the correlation coefficients of $\Phi_{o} / \Phi_{s}, \Phi_{c}$ and the FIP $_{\text {bias }}$ have the same sign in all three periods of time. The magnitude of the correlation coefficients is nearly the same during the time intervals of 1991-1992 and 1997-1999. It gets smaller during the time interval of 2003-2005. In all cases the probability of the null hypothesis is low.

Completely different results are found for the correlation coefficients of the total surface flux $\Phi_{s}$ and the $\mathrm{FIP}_{\text {bias }}$. The correlation coefficients have different signs and magnitude at three different time intervals. Moreover, the probabilities of the null hypothesis are higher than for the correlation coefficients of $\Phi_{o} / \Phi_{s}, \Phi_{c}$ and the $\mathrm{FIP}_{\text {bias }}$, showing that for the period 1991-1992 the total surface flux $\Phi_{s}$ and the FIP bias are likely uncorrelated. Therefore the relationship between the smoothed time-series of the parameter $\Phi_{s}$ and the $\operatorname{FIP}_{\text {bias }}$ is due to their solar cycle variations.

For the unified set of data, that contains points of those three set, the Spearman rank correlation of the $\mathrm{FIP}_{\text {bias }}$ and $\Phi_{o} / \Phi_{s}$ is 0.63 with the null hypothesis probability of $10^{-4}$. The same parameters for the correlation FIP $_{\text {bias }}$ and $\Phi_{c}$ are -0.54 and $10^{-3}$, respectively. For the unified set of data the correlation coefficient between $\Phi_{s}$ and the FIP bias is -0.48 and the null hypothesis probability is $10^{-4}$. As we see before this does not mean the causal connection between them.

The magnitude of the FIP $_{\text {bias }}$ was about of 1.5 factor higher in maximum of the 22 solar cycle than in the maximum of the 23 cycle. Results of Brooks et al. (2017) shows $\mathrm{FIP}_{\text {bias }} \approx-0.6$ near to the maximum of the cycle 24 . Following to SIDS sunspot data center (http://sidc.be/silso) the 24-th solar cycle was considerably lower than cycles 22 and 23 . Therefore the obtained maximum magnitude of the FIP $_{\text {bias }}$ is likely underestimated in comparison with results of Brooks et al. (2017). This can be expected because the chosen procedure of the data reduction does not fully reproduce the full-Sun measurements of the coronal FIP effect. Preliminary, we conclude that the coronal FIP fractionation can be related to the topological properties of the large-scale magnetic field of the Sun. The connection of the coronal FIP fractionation with activity cycle 


\begin{tabular}{ccccccc}
\hline & \multicolumn{2}{c}{$1991-1992$} & \multicolumn{2}{c}{$1997-1999$} & \multicolumn{2}{c}{$2003-2005$} \\
\hline & $\mathrm{r}$ & $\mathrm{p}$ & $\mathrm{r}$ & $\mathrm{p}$ & $\mathrm{r}$ & $\mathrm{p}$ \\
\hline $\mathrm{FIP}_{\text {bias }}$ vs $\Phi_{o} / \Phi_{s}$ & 0.72 & 0.002 & 0.71 & 0.001 & 0.44 & 0.026 \\
\hline $\mathrm{FIP}_{\text {bias }}$ vs $\Phi_{c}$ & -0.578 & 0.023 & -0.72 & 0.001 & -0.276 & 0.181 \\
\hline $\mathrm{FIP}_{\text {bias }}$ vs $\Phi_{s}$ & -0.06 & 0.82 & -0.66 & 0.01 & 0.46 & 0.02 \\
\hline
\end{tabular}

Table 4: The Spearman's rank correlations, $r$, and estimations of the probability of the null hypothesis, p, for the three particular periods of time.

is caused by the solar cycle variations of the topological characteristics of the solar magnetic fields.

\subsection{Stellar magnetic activity and FIP bias}

Figure $5 \mathrm{a}$ and $\mathrm{b}$ shows the FIP $_{\text {bias }}$ vs the calculated magnetic parameters $P_{A X}$ and $\overline{B^{(T)}}$ for our sample of stars including the smoothed solar cycle timeseries and data sets for the individual solar rotations (same as in Figure 4). Variations of the mean strength of the nonaxisymmetric toroidal magnetic field, $\overline{B^{(T)}}$, show a high level of scattering in the range from a few Gauss in solar case to hundreds Gauss in case of AB Dor and the M-dwarfs stars. Also, the solar toroidal magnetic field is significantly smaller than in the young solar analogs like $\pi^{1}$ Uma or $\chi^{1}$ Ori. Those stars have the higher rotation rates and the higher magnetic activity (Baliunas et al., 1995$)$. The partially convective solartype stars have the negative $\mathrm{FIP}_{\text {bias }}$ (Wood et al., 2012). The FIP bias shows some tendency to decrease with the increase of $\overline{B^{(T)}}$ in direction of the solar cycle variations. If we take into account the data sets for the individual solar rotations and restrict ourselves with the case of $\mathrm{FIP}_{\text {bias }}<0$, the Spearman rank correlation of the $\mathrm{FIP}_{\text {bias }}$ vs $\overline{B^{(T)}}$ will be $r=-0.2$ and the probability of the null hypothesis is $p=0.04$. The quite high significance level of this correlation is solely because of including the solar datasets. If we restrict the solar data by the mean point given in Table 2 , i.e., $\mathrm{FIP}_{\text {bias }}=-0.3$ and $\overline{B^{(T)}} \approx 1 \mathrm{G}$, we get $r=-0.03$ and $p=0.89$. This means that the data are likely uncorrelated. Therefore the results about the average correlation between FIP $_{\text {bias }}$ and $\overline{B^{(T)}}$ are likely not tenable if the magnetic cycle variations are not taken into account.

We tried to take into account the magnetic periodicity of some of the stars from our sample. Using results of Messina and Guinan (2003); Metcalfe et al. (2013) and Egeland (2017) we put the determined periods in the Table|2 . Comparing the time-series of the magnetic activities given by Metcalfe et al. (2013) we find that epoch of observation of the coronal chemical composition for the $\epsilon$ Eri, that is the young K2-dwarf, corresponds to the epoch of the low activity of the star. In Figures 5 (a) and (b) the points of the magnetic measurement which is the closest to that phase of the activity of $\epsilon$ Eri is marked by the yellow color. The same conclusion can be drawn for EK Dra. To determine the approximate phase of the magnetic activity we use results of Järvinen et al. (2007) and 
Telleschi et al. (2005). We found that measurements of the FIP $_{\text {bias }}$ are related to an epoch of the relatively low activity of the EK Dra. Using these hints and results about the solar cycle variations of the $\mathrm{FIP}_{\text {bias }}$ we can assume that for the other epochs of the magnetic measurements of the $\epsilon$ Eri the $\mathrm{FIP}_{\text {bias }} \leq-0.06$ and $\mathrm{FIP}_{\text {bias }}<-0.3$ for the EK Dra. Taking this into account may reduce the scattering on the parameter $\overline{B^{(T)}}$ in our sample of stars.

The AB Dor and the M-dwarfs stars form a group at the up-right corner of that Figure showing the high level of the toroidal magnetic field and the positive FIP $_{\text {bias }}$. Note that the AB Dor belongs to the T Tauri branch of cool stars which are on the pre-main sequence stage of evolution (Lalitha et al., 2013).

Figure 5b shows the $\mathrm{FIP}_{\text {bias }}$ vs the parameter $P_{A X}$ (see, Ed6) The $P_{A X}$ shows the relative contribution of the axisymmetric poloidal field on the stellar surface. On the Sun the given parameter is well correlated with ratio of the open and closed unsigned magnetic flux $\Phi_{o} / \Phi_{s}$. The the solar-type stars show the decrease of FIP bias with a decrease of $P_{A X}$. It is important that the given tendency goes in the same direction as the solar cycle variations of the FIP effect. If we take into account the data sets for the individual solar rotations and restrict ourselves with the case of $\mathrm{FIP}_{\text {bias }}<0$, the Spearman rank correlation of the $\mathrm{FIP}_{\text {bias }}$ and $P_{A X}$ will be $r=0.4$ and the probability of the null hypothesis is $p=0$. Excluding the data for the individual solar rotations, we get $r=0.3$ and $p=0.26$. Therefore the correlation between parameters of the coronal FIP fractionation and topology of magnetic activity for the solar-type stars is more robust than for the correlation of the $\mathrm{FIP}_{\text {bias }}$ vs $\overline{B^{(T)}}$. Similarly as above, using arguments of the magnetic periodicity of the solar-type stars the significance of correlation between the FIP bias and $P_{A X}$ can be increased.

The up-right corner of the $5 \mathrm{a}$ is occupied by a small sample of M-dwarf stars. Those stars show a relatively high amplitude of the poloidal magnetic field (see Table 2). This results in the high parameter $\mathrm{P}_{A X}$ in that sample. The topology of the large-scale magnetic field of for some of our M-dwarfs (e.g., EQ PegB) resembles the Sun's magnetic field during the cycle minims, having very strong dipole component of the large-scale magnetic field (Donati et al., 2008a). Those stars have the poloidal magnetic field of strength $1 \mathrm{kG}$ and more (Donati) et al. 2008a Morin et al., 2008). It is likely that the large-scale dynamo on those stars operates in a different way (for details, see, Morin et al. 2011; Pipin 2017, Pipin and Yokoi 2017). The M-dwarfs show the so-called "inverse" FIP ${ }_{\text {bias }}$ (Wood et al. [2012). The "inverse" FIP bias is also found on the AB Dor which is a solar analog passing the so-called T-Tauri stage of evolution (Lalitha et al. 2013). Interesting that ratio between the mean magnitudes of the magnetic field components $\left(\mathrm{P}_{A X}\right)$ on the $\mathrm{AB}$ Dor corresponds to that of others young solar analogs in our sample. On the other hand the FIP bias is similar to that found in M-dwarfs and similarly to M-dwarfs, the AB Dor shows rather a strong mean density of the poloidal magnetic field.

The exceptional properties of AB Dor are rather interesting. On one hand, we see that topology of the magnetic field of the star is similar to the solar-like stars sample. On another hand, the coronal FIP fractionation on AB Dor likely 

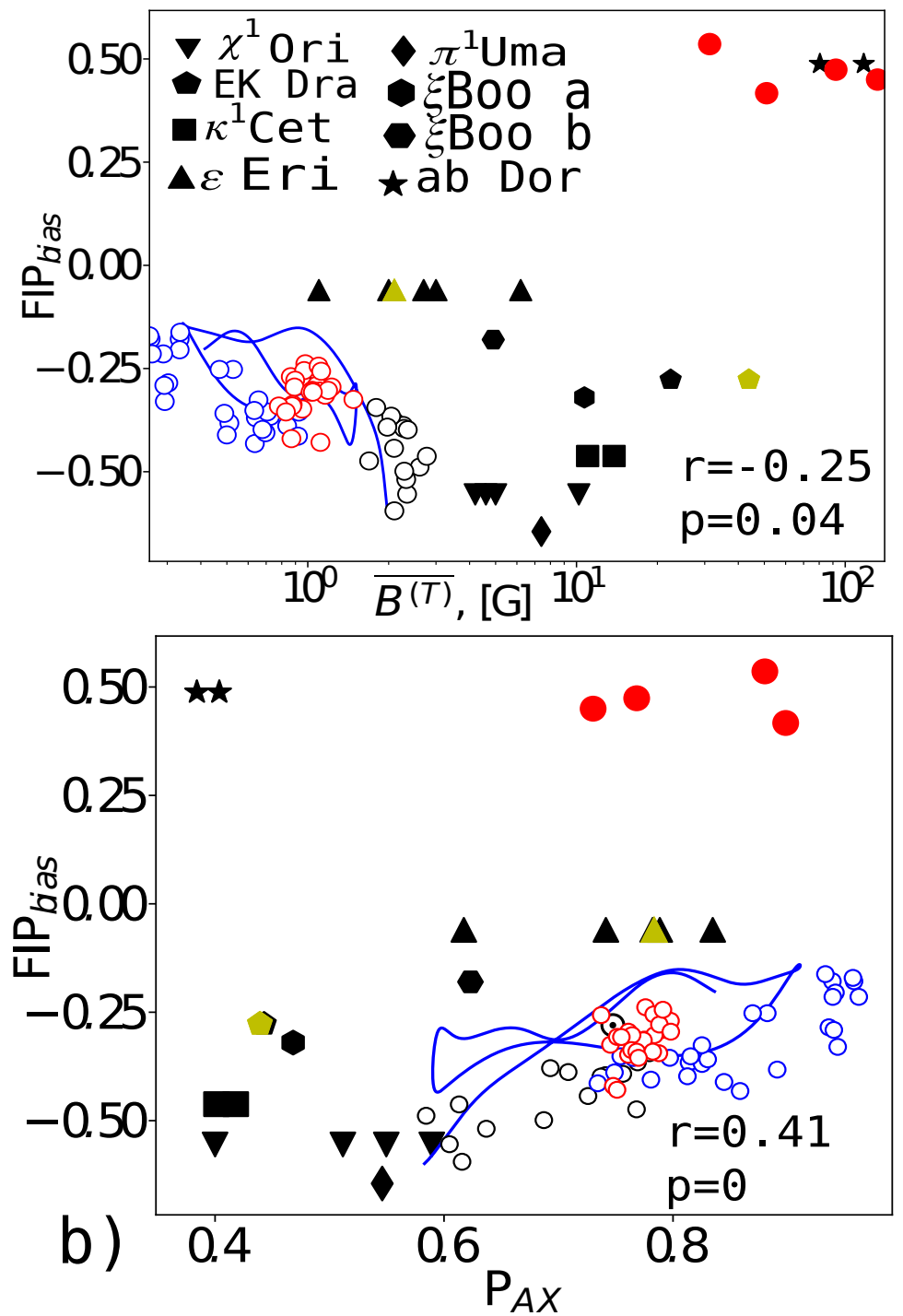

Figure 5: a) The FIP bias vs $\overline{B^{(T)}}$, the solar-cycle smoothed time series is shown by the solid line, the data for the individual rotations are shown by circles of the same color as in Figure 4 the red-filled circles show the M-dwarfs stars, the yellow marks stand for the magnetic observations which are close to epochs of measurements of FIP bias $_{\text {for }}$ ftars $\varepsilon$ Eri and EK Dra (see, the main text), $\mathrm{r}$ is the Spearman rank correlation and $\mathrm{p}$ is the probability of the null hypothesis; b) the same as (a) for the FIP bias vs $P_{A X}$ 
happens in a way similar to that demonstrated by the M-dwarfs. From our data (Table 2 we also can see that magnitude of the poloidal magnetic field is growing with increasing index B-V (associated with the decreasing stellar mass). This is in agreement with Donati et al. (2008a), Morin et al. (2008) and See et al. (2016). Results of stellar magnetic observations show that on the M-dwarfs the large-scale poloidal magnetic field forms the stellar coronal activity. The lack of the observational data does not allow to discuss a relation between magnetic activity and the FIP effect on M-dwarfs. Currently, we can only say that the topological properties of the magnetic activity on M-dwarfs are very different from the solar-type stars. On another hand results of Wright and Drake (2016) shows that all low main-sequence stars, including the solar-type stars and fully convective M-dwarfs, show the same relation of the X-ray luminosity on the convection Rossby number.

\section{Discussion and conclusions}

Findings of the paper should be considered as preliminary because of several reasons. There are two major issues in our analysis. Firstly, the time-averaged FIP effect deduced from the Ulysses dataset can not fully substitute the Sun as a star FIP-effect determined via spectroscopic methods (cf, Brooks et al., 2017 ). The cross-calibration between the in-situ measurements of the solar wind composition and the full-Sun spectroscopic observations can help to resolve this issue. Secondly, the introduced parameters of the unsigned open and closed magnetic flux are found very suitable for studying the solar and stellar wind (Jardine et al., 2017). These parameters may depend on the parameters of the source surface. Therefore the usability of the correlations shown in Figure 3 for the stellar activity analysis can be questioned because the radius of the source surface may depend on both the spectral type and age of a star. Also, the data set of the our stellar sample is rather small.

Keeping in mind the above issues the main results of the paper can be formulated as follow. The solar cycle variations of the FIP bias show a linear relationship to the ratio of the open and closed magnetic flux of the Sun as a star (see, Figur 44a). Taking into account results of Brooks et al. 2017 we see that the Sun as a star FIP $_{\text {bias }}$ keeps running in correlation with parameters $\Phi_{o} / \Phi_{s}$ and $P_{A X}$, varying in the range between -0.6 to -0.2 from cycle 22 to cycle 24 . It is shown that the correlation between the $\mathrm{FIP}_{\text {bias }}$ and $\Phi_{o} / \Phi_{s}$ exists both for the individual solar rotations and for the solar cycle variation of the Sun as a star FIP - effect. Also, there is a correlation between the FIP $_{\text {bias }}$ and $\Phi_{c}$ ( Figure4 3 ). For the individual solar rotations the statistical significance of relationship between $\mathrm{FIP}_{\text {bias }}$ and $\Phi_{c}$ is worse than for $\mathrm{FIP}_{\text {bias }}$ vs $\Phi_{o} / \Phi_{s}$. However, our results show that correlation of $\Phi_{c}$ and the FIP $_{\text {bias }}$ keep the same sign in all analyzed intervals of the solar cycle. The closed magnetic flux correlates with the mean toroidal magnetic field on the solar surface (see, Figur£3 $\mathrm{p}$ ). The large-scale toroidal magnetic field is often considered as the main source of the sunspot activity (Parker, 1955). Brooks et al. (2017) found a correlation between the Sun as a star FIP-effect and F10.7 radio flux. The latter is 
a good proxy for the sunspot activity. We conclude that the origin of the solar coronal FIP composition depends both on the topological characteristics of the large-scale solar coronal magnetic field and the level of the magnetic activity. The parameter $1-\Phi_{o} / \Phi_{s}$ measures the relative contribution of the closed magnetic configurations to the total unsigned magnetic flux. The parameter $\Phi_{c}$ characterize the surface-averaged strength of magnetic activity provided by the closed magnetic loops of the solar active regions. This averaging may reduce correlation coefficient for the relationship between $\mathrm{FIP}_{\text {bias }}$ and $\Phi_{c}$ in compare to $\mathrm{FIP}_{\text {bias }}$ and $\Phi_{o} / \Phi_{s}$. This issue needs to be studied further. Both parameters, the $\Phi_{o} / \Phi_{s}$ and $\Phi_{c}$ vary periodically with the solar cycle. This cause the solar cycle variation of the Sun as a star FIP - effect.

To investigate the stellar FIP - effect in the same way as the solar case, we introduce two tracers $P_{A X}$ and $\overline{B^{(T)}}$. Figure 3 and Table 3 show that these tracers are uniquely related with $\Phi_{o} / \Phi_{s}$ and $\Phi_{c}$. We employ this in the analysis of the stellar data set. Similar to the Sun, the solar-type stars show the increase of the stellar FIP bias goes with the increase of $P_{A X}$ (c.f., $\Phi_{o} / \Phi_{s}$ for the Sun). Correlation of the FIP $\mathrm{Fias}_{\text {bis }}$ to the magnitude of the toroidal magnetic field, $\overline{B^{(T)}}$ (cf, $\Phi_{c}$ for the Sun), is weak. This can be a result of the high magnetic variability in the young solar analogs (Baliunas et al. 1995). The additional observations of the FIP effect for the different stage of the stellar magnetic activity can help to clarify the results about the dependence of the $\mathrm{FIP}_{\text {bias }}$ with the magnetic activity cycles. The relationship between the stellar FIP bias to the level of the Xray luminosity of the stellar coronae remains contradictory. García-Alvarez et al. (2009) found a correlation between the normalized X-ray luminosity $\left(L_{\mathrm{X}} / L_{\mathrm{bol}}\right)$ and FIP on the late-type stars. However analysis of Wood et al. (2012), on the same sample of stars, had shown that this correlation is likely because of the relationship between the stellar FIP $_{\text {bias }}$ and the spectral class. They did not find a meaningful correlation of the stellar FIP bias and the level of the X-ray luminosity $\left(L_{X}\right)$ of the stellar coronae. Pevtsov et al. (2003) showed the highlevel significance correlation between the surface magnetic flux and the X-ray luminosity of the low main-sequence stars. The large-scale organization of the magnetic activity changes with age and with the spectral class of a star (Donati et al., 2008a, Reiners and Basri, 2009; See et al., 2016). Summarizing the above facts, we confirm our conclusions that in the solar analogs the magnitude of the FIP $_{\text {bias }}$ is connected both with the magnetic variability of stars and topology of the coronal magnetic field.

The above conclusion can be suitable for the theoretical scenario of the solar FIP effect which was developed by Laming (2015)(hereafter L15). Comparing to other ideas, e.g., Schwadron et al. (1999) (also, see the review of Tomozov, 2013), the scenario of L15 has an advantage explaining both the normal (solarlike) and the inversed FIP effect from the same mechanism. The key idea behind his model is that the FIP fractionation is induced by the so-called ponderomotive force. This force results from propagation of resonant alfvenic waves along the closed magnetic field lines. Direction and amplitude of the ponderomotive force depend on the gradient of wave energy in the low corona. For the resonant 
waves the energy grows up and this results in the upward ponderomotive force acting on ions of the low FIP. In the open magnetic field structures and for the off-resonant waves the wave energy decreases in the major part of the low corona (see Laming, 2004). Strong reflections of waves from upper boundary of transition region results in inversion of the ponderomotive force and inversion of the FIP effect. The detailed calculations made by Wood et al. (2012) and Laming(2015) demonstrated the efficiency of the given mechanism both for the Sun and the M-dwarfs stars. Note, that the solar relationship between the $\mathrm{FIP}_{\text {bias }}$ and $\Phi_{o} / \Phi_{s}$ can be also fitted in the model of Schwadron et al. (1999). They suggested that FIP fractionation is developed due to the wave heating of minor ions in on the closed configurations of large coronal loops. However, their theory does not explain the inversed FIP effect.

The given scenario gives a natural explanation for the solar cycle relation between parameters of the open and close magnetic flux and the coronal FIP $\mathrm{Fias}_{\text {sia }}$ for the Sun as a star. Note that the alfvenic waves are one of the possible mechanism for the solar corona heating (Aschwanden, 2005). Interesting that estimations of L15 showed that magnitude of the density energy of alfvenic waves is the one that it is necessary for to maintain the magnitude of the $\mathrm{FIP}_{\text {bias }}$ in the magnetic arcade. This density energy of alfvenic waves corresponds to that needed for the coronal heating, as well. The mechanism suggested by L15 was never applied to estimate the $\mathrm{FIP}_{\text {bias }}$ for the Sun as a star. Also, the linear estimations of the ponderomotive force profiles given by Laming (2015) can be inconsistent as we have to take into account the nonlinear effects (see.Shestov et al., 2017).

Laming (2004) and Wood et al. (2012) argued that the inverse FIP $_{\text {bias }}$ on M-dwarfs can be related with strong reflections of alfvenic waves from the upper boundary of the transition region. The effect of strong reflections can be caused by difference of the vertical scales of transition region at the base of the opposite legs of the magnetic arcades (see, Laming, 2004 and Hollweg, 1984). This is expected to find out on active M-dwarfs. Those stars show magnetic field organization which consists of the large-scale patches of strong magnetic field (Donati et al., 2008a).

Roughly speaking the inverse FIP $_{\text {bias }}$ on M-dwarfs can be related to the dramatic increase of magnitude of the coronal magnetic field. The given mechanisms are considered as the theoretical scenarios which need the further development.

Our conclusions can be summarized as follows. The solar-like FIP fractionation of the coronal elements is likely related to the topology of the large-scale coronal magnetic field and activity level of the toroidal magnetic field. The FIP $_{\text {bias }}$ of the Sun as a star varies in the solar cycle in inverse proportion to the contribution of the unsigned closed magnetic flux, which depends on ratio the mean strength of the large-scale poloidal and toroidal magnetic field on the solar surface. Available theoretical scenarios suggest that the solar-like FIP $_{\text {bias }}$ as well as the inverse FIP $_{\text {bias }}$ can be described in the unified picture relating effects of the large-scale the topology of magnetic fields and the nonlinear processes of coronal waves propagation. 
Acknowledgments. This work was conducted as a part of FR program II.16 of ISTP SB RAS. VP thanks the financial support from of RFBR grant $17-52-53203$.

\section{REFERENCES}

\section{References}

Aschwanden, M. J., Dec. 2005. Physics of the Solar Corona. An Introduction with Problems and Solutions (2nd edition).

Baker, D., Brooks, D. H., Démoulin, P., Yardley, S. L., van Driel-Gesztelyi, L., Long, D. M., Green, L. M., Apr. 2015. FIP Bias Evolution in a Decaying Active Region. ApJ802, 104.

Baliunas, S. L., Donahue, R. A., Soon, W. H., Horne, J. H., Frazer, J., WoodardEklund, L., Bradford, M., Rao, L. M., Wilson, O. C., Zhang, Q., Bennett, W., Briggs, J., Carroll, S. M., Duncan, D. K., Figueroa, D., Lanning, H. H., Misch, T., Mueller, J., Noyes, R. W., Poppe, D., Porter, A. C., Robinson, C. R., Russell, J., Shelton, J. C., Soyumer, T., Vaughan, A. H., Whitney, J. H., Jan. 1995. Chromospheric variations in main-sequence stars. ApJ438, 269-287.

Brooks, D., Baker, D., van Driel-Gesztelyi, L., Warren, H., 2017. A solar cycle correlation of coronal element abundances in sun-as-a-star observations. Nature Communications 8, 183.

URL https://doi.org/10.1038/s41467-017-00328-7

Buergi, A., Geiss, J., Feb. 1986. Helium and minor ions in the corona and solar wind - Dynamics and charge states. Sol.Phys.103, 347-383.

Donati, J.-F., 2001. Imaging the Magnetic Topologies of Cool Active Stars. In: Boffin, H. M. J., Steeghs, D., Cuypers, J. (Eds.), Astrotomography, Indirect Imaging Methods in Observational Astronomy. Vol. 573 of Lecture Notes in Physics, Berlin Springer Verlag. p. 207.

Donati, J.-F., Brown, S. F., Oct. 1997. Zeeman-Doppler imaging of active stars. V. Sensitivity of maximum entropy magnetic maps to field orientation. A \& A326, 1135-1142.

Donati, J.-F., Jardine, M. M., Petit, P., Morin, J., Bouvier, J., Collier Cameron, A., Delfosse, X., Dintrans, B., Dobler, W., Dougados, C., Ferreira, J., Forveille, T., Gregory, S. G., Harries, T., Hussain, G. A. J., Menard, F., Paletou, F., Apr. 2008a. Magnetic Topologies of Cool Stars. In: van Belle, G. (Ed.), 14th Cambridge Workshop on Cool Stars, Stellar Systems, and the Sun. Vol. 384 of Astronomical Society of the Pacific Conference Series. p. 156.

Donati, J.-F., Landstreet, J. D., Sep. 2009. Magnetic Fields of Nondegenerate Stars. An. Rev. Astron. Astroph.47, 333-370. 
Donati, J.-F., Morin, J., Petit, P., Delfosse, X., Forveille, T., Aurière, M., Cabanac, R., Dintrans, B., Fares, R., Gastine, T., Jardine, M. M., Lignières, F., Paletou, F., Ramirez Velez, J. C., Théado, S., Oct. 2008b. Large-scale magnetic topologies of early M dwarfs. MNRAS390, 545-560.

Duvall, Jr., T. L., Scherrer, P. H., Svalgaard, L., Wilcox, J. M., Mar. 1979. Average photospheric poloidal and toroidal magnetic field components near solar minimum. Sol.Phys.61, 233-245.

Egeland, R., 2017. Long-Term Variability of the Sun in the Context of SolarAnalog Stars. Ph.D. thesis.

Fletcher, L., Cargill, P. J., Antiochos, S. K., Gudiksen, B. V., May 2015. Structures in the Outer Solar Atmosphere. Space Sci. Rev.188, 211-249.

García-Alvarez, D., Drake, J. J., Testa, P., Feb. 2009. Neon and Chemical Fractionation Trends in Late-type Stellar Atmospheres. In: Stempels, E. (Ed.), 15th Cambridge Workshop on Cool Stars, Stellar Systems, and the Sun. Vol. 1094 of American Institute of Physics Conference Series. pp. 796-799.

Geiss, J., Gloeckler, G., von Steiger, R., Apr. 1995. Origin of the Solar Wind From Composition Data. Space Sci. Rev.72, 49-60.

Hoeksema, J. T., Apr. 1995. The Large-Scale Structure of the Heliospheric Current Sheet During the ULYSSES Epoch. Space Sci. Rev.72, 137-148.

Hollweg, J. V., Feb. 1984. Resonances of coronal loops. ApJ277, 392-403.

Jardine, M., Vidotto, A. A., See, V., Feb. 2017. Estimating stellar wind parameters from low-resolution magnetograms. MNRAS465, L25-L29.

Järvinen, S. P., Berdyugina, S. V., Korhonen, H., Ilyin, I., Tuominen, I., Sep. 2007. EK Draconis. Magnetic activity in the photosphere and chromosphere. A \& A472, 887-895.

Lalitha, S., Fuhrmeister, B., Wolter, U., Schmitt, J. H. M. M., Engels, D., Wieringa, M. H., Dec. 2013. A multi-wavelength view of AB Doradus outer atmosphere . Simultaneous X-ray and optical spectroscopy at high cadence. A \& A560, A69.

Laming, J. M., Oct. 2004. A Unified Picture of the First Ionization Potential and Inverse First Ionization Potential Effects. ApJ614, 1063-1072.

Laming, J. M., Dec. 2015. The FIP and Inverse FIP Effects in Solar and Stellar Coronae. Living Reviews in Solar Physics 12, 2.

Liefke, C., Ness, J.-U., Schmitt, J. H. M. M., Maggio, A., Dec. 2008. Coronal properties of the EQ Pegasi binary system. A \& A491, 859-872. 
Marsden, S. C., Petit, P., Jeffers, S. V., Morin, J., Fares, R., Reiners, A., do Nascimento, J.-D., Aurière, M., Bouvier, J., Carter, B. D., Catala, C., Dintrans, B., Donati, J.-F., Gastine, T., Jardine, M., Konstantinova-Antova, R., Lanoux, J., Lignières, F., Morgenthaler, A., Ramìrez-Vèlez, J. C., Théado, S., Van Grootel, V., BCool Collaboration, Nov. 2014. A BCool magnetic snapshot survey of solar-type stars. MNRAS444, 3517-3536.

Messina, S., Guinan, E. F., Oct. 2003. Magnetic activity of six young solar analogues II. Surface Differential Rotation from long-term photometry. A \& A409, 1017-1030.

Mestel, L., 1968. Magnetic braking by a stellar wind-I. MNRAS138, 359.

Metcalfe, T. S., Buccino, A. P., Brown, B. P., Mathur, S., Soderblom, D. R., Henry, T. J., Mauas, P. J. D., Petrucci, R., Hall, J. C., Basu, S., Feb. 2013. Magnetic Activity Cycles in the Exoplanet Host Star epsilon Eridani. ApJL763, L26.

Meyer, J.-P., 1985. The baseline composition of solar energetic particles. ApJS57, 151-171.

Morin, J., Donati, J.-F., Petit, P., Delfosse, X., Forveille, T., Albert, L., Aurière, M., Cabanac, R., Dintrans, B., Fares, R., Gastine, T., Jardine, M. M., Lignières, F., Paletou, F., Ramirez Velez, J. C., Théado, S., Oct. 2008. Largescale magnetic topologies of mid M dwarfs. MNRAS390, 567-581.

Morin, J., Dormy, E., Schrinner, M., Donati, J.-F., Nov. 2011. Weak- and strong-field dynamos: from the Earth to the stars. MNRAS418, L133-L137.

Noyes, R. W., Weiss, N. O., Vaughan, A. H., Dec. 1984. The relation between stellar rotation rate and activity cycle periods. ApJ287, 769-773.

Parker, E., 1955. Hydromagnetic dynamo models. Astrophys. J. 122, 293-314.

Petit, P., Louge, T., Théado, S., Paletou, F., Manset, N., Morin, J., Marsden, S. C., Jeffers, S. V., May 2014. PolarBase: A Database of High-Resolution Spectropolarimetric Stellar Observations. Publications of the Astron. Soc. of Pacific126, 469.

Pevtsov, A. A., Fisher, G. H., Acton, L. W., Longcope, D. W., Johns-Krull, C. M., Kankelborg, C. C., Metcalf, T. R., Dec. 2003. The Relationship Between X-Ray Radiance and Magnetic Flux. ApJ598, 1387-1391.

Pipin, V., Tomozov, V., 2018. The nature of variations in anomalies of the chemical composition of the solar corona with the 11-year cycle. Astronomy Reports 62, 281-287.

Pipin, V. V., Apr. 2017. Non-linear regimes in mean-field full-sphere dynamo. MNRAS466, 3007-3020. 
Pipin, V. V., Pevtsov, A. A., Jul. 2014. Magnetic Helicity of the Global Field in Solar Cycles 23 and 24. ApJ789, 21.

Pipin, V. V., Yokoi, N., Dec. 2017. Generation of large-scale magnetic field in convective full-sphere cross-helicity dynamo. ArXiv e-prints.

Pottasch, S. R., 1963. The lower solar corona: the abundance of iron. MNRAS125, 543.

Reiners, A., Dec. 2012. Observations of Cool-Star Magnetic Fields. Living Reviews in Solar Physics 9, 1.

Reiners, A., Basri, G., Mar. 2009. On the magnetic topology of partially and fully convective stars. A \& A496, 787-790.

Rosén, L., Kochukhov, O., Hackman, T., Lehtinen, J., Sep. 2016. Magnetic fields of young solar twins. A \& A593, A35.

Schatten, K. H., Wilcox, J. M., Ness, N. F., Mar. 1969. A model of interplanetary and coronal magnetic fields. Sol.Phys.6, 442-455.

Schwadron, N. A., Fisk, L. A., Zurbuchen, T. H., Aug. 1999. Elemental Fractionation in the Slow Solar Wind. ApJ521, 859-867.

See, V., Jardine, M., Vidotto, A. A., Donati, J.-F., Boro Saikia, S., Bouvier, J., Fares, R., Folsom, C. P., Gregory, S. G., Hussain, G., Jeffers, S. V., Marsden, S. C., Morin, J., Moutou, C., do Nascimento, J. D., Petit, P., Waite, I. A., Nov. 2016. The connection between stellar activity cycles and magnetic field topology. MNRAS462, 4442-4450.

See, V., Jardine, M., Vidotto, A. A., Donati, J.-F., Folsom, C. P., Boro Saikia, S., Bouvier, J., Fares, R., Gregory, S. G., Hussain, G., Jeffers, S. V., Marsden, S. C., Morin, J., Moutou, C., do Nascimento, J. D., Petit, P., Rosén, L., Waite, I. A., Nov. 2015. The energy budget of stellar magnetic fields. MNRAS453, 4301-4310.

Shestov, S. V., Nakariakov, V. M., Ulyanov, A. S., Reva, A. A., Kuzin, S. V., May 2017. Nonlinear Evolution of Short-wavelength Torsional Alfvén Waves. ApJ840, 64 .

Skumanich, A., Feb. 1972. Time Scales for CA II Emission Decay, Rotational Braking, and Lithium Depletion. ApJ171, 565.

Telleschi, A., Güdel, M., Briggs, K., Audard, M., Ness, J.-U., Skinner, S. L., Mar. 2005. Coronal Evolution of the Sun in Time: High-Resolution X-Ray Spectroscopy of Solar Analogs with Different Ages. ApJ622, 653-679.

Testa, P., Drake, J. J., Peres, G., Dec. 2004. The Density of Coronal Plasma in Active Stellar Coronae. ApJ617, 508-530. 
Tomozov, V., 2013. On regularities of distribution of chemical composition in atmospheres of stars. Solnecho-zemnaya fizika 23, 23-32.

Vidotto, A. A., Jun. 2016. The magnetic field vector of the Sun-as-a-star. MNRAS459, 1533-1542.

Vidotto, A. A., Gregory, S. G., Jardine, M., Donati, J. F., Petit, P., Morin, J., Folsom, C. P., Bouvier, J., Cameron, A. C., Hussain, G., Marsden, S., Waite, I. A., Fares, R., Jeffers, S., do Nascimento, J. D., Jul. 2014. Stellar magnetism: empirical trends with age and rotation. MNRAS441, 2361-2374.

von Steiger, R., Geiss, J., Gloeckler, G., Galvin, A. B., Apr. 1995. Kinetic Properties of Heavy Ions in the Solar Wind From SWICS/Ulysses. Space Sci. Rev.72, 71-76.

Wang, Y.-M., Nov. 2012. Semiempirical Models of the Slow and Fast Solar Wind. Space Sci. Rev.172, 123-143.

Warren, H. P., May 2014. Measurements of Absolute Abundances in Solar Flares. ApJL786, L2.

Warren, H. P., Brooks, D. H., Doschek, G. A., Feldman, U., Jun. 2016. Transition Region Abundance Measurements During Impulsive Heating Events. ApJ824, 56.

Widing, K. G., Feldman, U., Jul. 2001. On the Rate of Abundance Modifications versus Time in Active Region Plasmas. ApJ555, 426-434.

Woo, R., Habbal, S. R., Feldman, U., Sep. 2004. Role of Closed Magnetic Fields in Solar Wind Flow. ApJ612, 1171-1174.

Wood, B. E., Laming, J. M., Karovska, M., Jul. 2012. The Coronal Abundance Anomalies of M Dwarfs. ApJ753, 76.

Wood, B. E., Linsky, J. L., May 2006. Coronal Emission Measures and Abundances for Moderately Active K Dwarfs Observed by Chandra. ApJ643, 444459 .

Wood, B. E., Linsky, J. L., Jul. 2010. Resolving the $\xi$ Boo Binary with Chandra, and Revealing the Spectral Type Dependence of the Coronal "FIP Effect". ApJ717, 1279-1290.

Wright, N. J., Drake, J. J., Jul. 2016. Solar-type dynamo behaviour in fully convective stars without a tachocline. Nature535, 526-528.

Zurbuchen, T. H., Weberg, M., von Steiger, R., Mewaldt, R. A., Lepri, S. T., Antiochos, S. K., Jul. 2016. Composition of Coronal Mass Ejections. ApJ826, 10. 